\title{
A Note on an Iterative Method for Generalized Inversion of Matrices*
}

\section{By Adi Ben-Israel}

The iterative method of Schulz [4], [3] for matrix inversion was generalized in [1] as follows:

THEOREM 1. The sequence of matrices defined by

$$
X_{k+1}=X_{k}\left(2 P_{R(A)}-A X_{k}\right) \quad(k=0,1, \cdots)
$$

where $X_{0}$ is an $n \times m$ complex matrix satisfying

$$
\begin{aligned}
X_{0} & =A^{*} B_{0}, \quad B_{0} \text { some nonsingular } m \times m \text { matrix, } \\
X_{0} & =C_{0} A^{*}, \quad C_{0} \text { some nonsingular } n \times n \text { matrix, } \\
\left\|A X_{0}-P_{R(A)}\right\| & <1, \quad(\|\| \text { any matrix norm }[3]), \\
\left\|X_{0} A-P_{R\left(A^{*}\right)}\right\| & <1,
\end{aligned}
$$

converges to the generalized inverse $A^{+}$of $A$.

As pointed out in [1], the computational significance of the method (1) is limited by the need for knowledge of $P_{R(A)}$ (and of $P_{R\left(A^{*}\right)}$ if condition (5) is to be checked). This difficulty is evaded in the following theorem.

Theorem 2. Let $A$ be an arbitrary (nonzero) complex $m \times n$ matrix of rank $r$ and let

$$
\lambda_{1}\left(A A^{*}\right) \geqq \lambda_{2}\left(A A^{*}\right) \geqq \cdots \geqq \lambda_{r}\left(A A^{*}\right)
$$

denote the nonzero eigenvalues of $A A^{*}$. If the real scalar $\alpha$ satisfies

$$
0<\alpha<\frac{2}{\lambda_{1}\left(A A^{*}\right)}
$$

then the sequence defined by:

$$
\begin{aligned}
X_{0} & =\alpha A^{*} \\
X_{k+1} & =X_{k}\left(2 I-A X_{k}\right) \quad(k=0,1, \cdots) .
\end{aligned}
$$

converges to $A^{+}$as $k \rightarrow \infty$.

Proof. $X_{0}$ defined by (7), (6) satisfies (2), (3), (4) and (5). To prove that $X_{0}$ of $(7)$, (6) satisfies (4) we note that $A A^{+}\left(=P_{R(A)}\right)$ and $A A^{*}$ are commuting Hermitian matrices with the same range space. The eigenvalues of the $m \times m$ matrix: $A X_{0}-P_{R(A)}=\alpha A A^{*}-A A^{+}$are therefore

$$
\begin{cases}\alpha \lambda_{i}\left(A A^{*}\right)-1 & (i=1, \cdots, r) \\ 0 & (i=r+1, \cdots, m)\end{cases}
$$

Received August 5, 1965. Revised August 26, 1965.

* Research sponsored by the Office of Naval Research, Contract Nonr-1228(10), Project NR 047-021 and by the U.S. Army Research Office-Durham, Contract No. DA-31-124-ARO-D322 . Reproduction of this paper in whole or in part is permitted for any purpose of the United States Government. 
and, by (6), are all: $<1$ in absolute value:

$$
\left|\lambda_{i}\left(\alpha A A^{*}-A A^{+}\right)\right|<1 \quad(i=1, \cdots, m)
$$

similarly

$$
\left|\lambda_{i}\left(\alpha A^{*} A-A^{+} A\right)\right|<1 \quad(i=1, \cdots, n) .
$$

(Indeed the nonzero eigenvalues of $\left(\alpha A A^{*}-A A^{+}\right),\left(\alpha A^{*} A-A^{+} A\right)$ are identical.) With the lub S-norm [3, p. 44] in (4) and (5), both hold because of (10) and (11). (Actually (10) and (11) suffice for the convergence of (8).)

Now the process (1) initiated with: $X_{0}=\alpha A^{*}$ retains the form [1, Eq. (12)]:

$$
X_{k}=C_{k} A^{*} \quad(k=1,2, \cdots)
$$

and since

$$
A^{*} P_{R(A)}=A^{*}
$$

it follows that:

$$
X_{k}\left(2 P_{R(A)}-A X_{k}\right)=X_{k}\left(2 I-A X_{k}\right) \quad(k=0,1, \cdots)
$$

and the convergence of (8) follows from that of (1). Q.E.D.

Remarks.

a) Similarly, the sequence defined by

$$
X_{k+1}=\left(2 I-X_{k} A\right) X_{k} \quad(k=0,1, \cdots)
$$

with $X_{0}=\alpha A^{*}$, converges to $A^{+}$.

b) In using the method (8) it is not necessary to compute $\lambda_{1}\left(A A^{*}\right)$ : Writing

$$
A A^{*}=\left(b_{i j}\right) \quad(i, j=1, \cdots, m)
$$

we conclude from the Gershgorin theorem, [3] that:

$$
\lambda_{1}\left(A A^{*}\right) \leqq \max _{i=1, \cdots, m}\left\{\sum_{j=1}^{m}\left|b_{i j}\right|\right\} .
$$

Condition (6) can therefore be replaced, e.g. by

$$
0<\alpha<\frac{2}{\max _{i=1, \cdots, m}\left\{\sum_{j=1}^{m}\left|b_{i \jmath}\right|\right\}} .
$$

c) Examples and applications will be given in [2].

Technicon, Israel Institute of Technology

Haifa, Israel

Northwestern University

Evanston, Illinois

1. A. BEN-IsRAEL, "An iterative method for computing the generalized inverse of an arbitrary matrix," Math. of Comp., v. 19, 1965, pp. 452-455.

2. A. BEN-IsRael \& D. Cohen, "On iterative computation of generalized inverses and associated projections," J. Soc. Indust. Appl. Math. Ser. Numer. Anal. (To appear.) 1964 .

3. A. S. Housenolder, The Theory of Matrices in Numerical Analysis, Blaisdell, New York,

4. G. Schulz, "Iterative Berechnung der reziproken Matrix," Z. Angew. Math. Mech., v. 13, 1933, pp. 57-59. 